\title{
Jordan frame no-hair for spherical scalar-tensor black holes
}

\author{
Valerio Faraoni, ${ }^{1, *}$ \\ ${ }^{1}$ Physics Department, Bishop's University, 2600 College Street, Sherbrooke, Québec, Canada J1M 1Z7
}

\begin{abstract}
A no-hair theorem for spherical black holes in scalar-tensor gravity is presented. Contrary to the existing theorems, which are proved in the Einstein conformal frame, this proof is performed entirely in the Jordan frame. The theorem is limited to spherical symmetry (instead of axisymmetry) but holds for non-constant Brans-Dicke couplings.
\end{abstract}

\section{INTRODUCTION}

There is little doubt that general relativity (GR) should eventually merge with quantum mechanics at the Planck scale, but it is not presently known how this should be done, although there are contenders to the role of a quantum gravity theory. It seems pretty clear, however, that quantizing gravity introduces extra degrees of freedom in addition to the metric tensor of GR, such as scalar and $p$-form fields, higher derivative terms in the field equations, and other deviations from GR. What is more, the acceleration of the present cosmic expansion discovered with type Ia supernovae is explained in the context of the GR-based $\Lambda$ CDM model only by postulating a very exotic and completely ad hoc dark energy accounting for approximately $70 \%$ of the energy content of the universe, the origin of which is a complete mystery (see Refs. 11, 2] for reviews and references). A viable alternative to introducing dark energy is to modify gravity at cosmological scales [ 3 3 ], which has led to the popular class of $f(R)$ theories of gravity (see [6] for reviews). To add to these motivations, GR is tested poorly in many regimes and assuming its validity is currently more a leap of faith than an experimental fact 7, 8].

It is possible that some deviations from GR may be detectable at astrophysical scales involving black holes and strong gravity. For this reason, many theoretical efforts have focused on the possibility of detecting black hole hair, which is forbidden in (vacuum) GR [9, 10] but is allowed in other theories of gravity $11-13$. The JebsenBirkhoff theorem of GR [14, 15] states that the only vacuum, spherically symmetric, asymptotically flat solution of the Einstein equations with zero cosmological constant is the Schwarzschild solution. In addition to a host of nohair theorems in GR [9, 10], similar theorems have been proved also in certain other theories of gravity, including the theory of a scalar field non-minimally coupled to gravity, Brans-Dicke and scalar-tensor theories [16 21], and Horndeski gravity (see 11 13] for recent reviews). Here we focus on scalar-tensor theories of gravity, which include $f(R)$ gravity as a subclass [6]. A celebrated no-hair theorem by Hawking 18] states that all asymptotically flat, axisymmetric, stationary black holes in Brans-Dicke theory with constant Brans-Dicke coupling $\omega$ and zero

\footnotetext{
* vfaraoni@ubishops.ca
}

potential for the Brans-Dicke gravitational scalar field $\phi$ are GR (i.e., Kerr or Kerr-Newman) black holes. This result is important because these black holes represent the endpoint of gravitational collapse (indeed, the endpoints of collapse calculated numerically in Brans-Dicke gravity are invariably GR black holes [22]). This theorem has been generalized recently to include more general scalar-tensor theories in which the Brans-Dicke coupling becomes a function $\omega(\phi)$ of the scalar and there is a potential $V(\phi)$ [20]. The more recent Ref. 21] includes the case in which the black hole has de Sitter (instead of Minkowski) asymptotics.

There are, however, important limitations in these results: first, black holes can be perturbed from their stationary equilibrium state by surrounding matter, which can take the form of a star or of another black hole in a binary system, or of matter flowing in from a surrounding accretion disk. The resulting dynamics may give away some deviations from GR, but the no-hair theorems fail to catch this physics. Second, the assumption of asymptotic flatness is, at best, an approximation which may be good in practice, but ultimately realistic black holes are embedded in the universe. This universe does not have a de Sitter asymptotics, but a FriedmannLemaître-Robertson-Walker (FLRW) one. Therefore, although much effort has gone into establishing more general no-hair theorems or probing the scalar hair deviations from GR, there are still important gaps in our understanding of these issues.

Independent motivation for extending the available nohair theorems of scalar-tensor gravity comes from the fact that all these theorems thus far have been obtained in the Einstein conformal frame representation of the theory. While it is reasonable to regard the Jordan and the Einstein conformal frames as equivalent representations, as proposed long ago by Dicke 23], there has been much discussion as to whether these two frames are physically equivalent 24], and the debate has not settled. Part of the problem consists of defining what "physically equivalent" precisely means. We do not weigh in on this issue here but, for the skeptic, we provide a no-hair theorem which is entirely based in the Jordan frame. It extends Hawking's no-hair theorems by allowing for non-constant Brans-Dicke couplings, but it is restricted to spherical black holes, contrary to Hawking's result and its more recent generalizations 20, 21] which hold for more realistic axiysimmetric (i.e., rotating) black holes. Therefore, the value of our theorem lies not so much in extend- 
ing previous results (spherical symmetry is indeed a serious restriction), but rather in showing that the Jordan frame allows for essentially the same results that can be obtained in the Einstein frame, although the techniques used are quite different (we reason on the local field equations instead of using integrals of these equations over the horizon and at spatial infinity).

We begin by reviewing the essential features of scalartensor gravity, in the notation of 25]. Scalar-tensor theories are described by the action [26, 27]

$$
\begin{aligned}
S_{S T}= & \frac{1}{16 \pi} \int d^{4} x \sqrt{-g}\left[\phi R-\frac{\omega(\phi)}{\phi} g^{a b} \nabla_{a} \phi \nabla_{b} \phi\right. \\
& -V(\phi)]+S^{(m)}
\end{aligned}
$$

in the Jordan conformal frame, where $S^{(m)}$ is the matter part of the action, $R$ is the Ricci scalar, $\phi$ is the BransDicke-like scalar field, the function $\omega(\phi)$ is the BransDicke coupling, and $V(\phi)$ is a potential. The field equations obtained by varying (1.1) can be written in the form of effective Einstein equations as

$$
\begin{aligned}
R_{a b}-\frac{1}{2} g_{a b} R= & \frac{8 \pi}{\phi} T_{a b}^{(m)} \\
& +\frac{\omega(\phi)}{\phi^{2}}\left(\nabla_{a} \phi \nabla_{b} \phi-\frac{1}{2} g_{a b} \nabla^{c} \phi \nabla_{c} \phi\right) \\
& +\frac{1}{\phi}\left(\nabla_{a} \nabla_{b} \phi-g_{a b} \square \phi\right)-\frac{V(\phi)}{2 \phi} g_{a b} \\
\equiv & \frac{8 \pi}{\phi}\left(T_{a b}^{(m)}+T_{a b}^{(\phi)}\right), \\
(2 \omega+3) \square \phi= & 8 \pi T^{(m)}-\frac{d \omega}{d \phi} \nabla^{c} \phi \nabla_{c} \phi+\phi \frac{d V}{d \phi}-2 V
\end{aligned}
$$

where $T_{a b}^{(m)}$ is the matter energy-momentum tensor and we assume that $\phi>0$ in conjunction with $\omega>-3 / 2$ to guarantee the positivity of the effective gravitational coupling 28]

$$
G_{e f f}=\frac{2(\omega+2)}{(2 \omega+3) \phi} .
$$

By imposing that the matter energy-momentum tensor $T_{a b}^{(m)}$ vanishes, a time-dependent $T_{a b}^{(\phi)}$ would spoil the validity of the Jebsen-Birkhoff theorem and only the assumption that $\phi$ is time-independent (or that $T_{a b}^{(\phi)}$ is static, as for a time-dependent stealth field $\phi$ [29] or for other solutions which do not enjoy the symmetries of the metric [1]]), restores the staticity of spherical geometries.

When $\phi=$ const. $\equiv \phi_{0}$, Eq. (1.2) reduces to

$$
R_{a b}-\frac{1}{2} g_{a b} R=\frac{8 \pi}{\phi_{0}} T_{a b}^{(m)}-\frac{V_{0}}{2 \phi_{0}} g_{a b},
$$

where $V_{0} \equiv V\left(\phi_{0}\right)$ if a scalar field potential is present, hence the theory degenerates into GR with the cosmological constant $\Lambda \equiv V_{0} /\left(2 \phi_{0}\right)$.
By performing the conformal transformation of the metric and redefining non-linearly the Brans-Dicke-like scalar as in

$$
\begin{aligned}
& g_{a b} \rightarrow \tilde{g}_{a b}=\Omega^{2} g_{a b}, \quad \Omega=\sqrt{\phi}, \\
& d \tilde{\phi}=\sqrt{\frac{2 \omega(\phi)+3}{16 \pi}} \frac{d \phi}{\phi},
\end{aligned}
$$

the scalar-tensor action (1.1) assumes the Einstein frame form

$$
\begin{aligned}
S_{S T}= & \int d^{4} x \sqrt{-\tilde{g}}\left[\frac{\tilde{R}}{16 \pi}-\frac{1}{2} \tilde{g}^{a b} \tilde{\nabla}_{a} \tilde{\phi} \tilde{\nabla}_{b} \tilde{\phi}-U(\tilde{\phi})\right. \\
& \left.+\frac{\mathcal{L}^{(m)}}{\phi^{2}}\right],
\end{aligned}
$$

where a tilde denotes quantities associated with the rescaled metric $\tilde{g}_{a b}, \mathcal{L}^{(m)}$ is the matter Lagrangian density, and

$$
U(\tilde{\phi})=\frac{V[\phi(\tilde{\phi})]}{[\phi(\tilde{\phi})]^{2}} .
$$

This is formally the action of GR with a minimally coupled scalar field $\tilde{\phi}$ but with the important difference that this scalar now couples explicitly to matter [23]. The Einstein frame field equations are

$$
\begin{aligned}
& \tilde{R}_{a b}-\frac{1}{2} \tilde{g}_{a b} \tilde{R}=\frac{8 \pi}{\phi^{2}} T_{a b}^{(m)}+8 \pi \tilde{T}_{a b}^{(\tilde{\phi})}, \\
& \tilde{\square} \tilde{\phi}-\frac{d U}{d \tilde{\phi}}=\frac{8 \pi T^{(m)}}{\phi^{2}},
\end{aligned}
$$

where

$$
\tilde{T}_{a b}^{(\tilde{\phi})}=\tilde{\nabla}_{a} \tilde{\phi} \tilde{\nabla}_{b} \tilde{\phi}-\frac{1}{2} \tilde{g}_{a b} \tilde{g}^{c d} \tilde{\nabla}_{c} \tilde{\phi} \tilde{\nabla}_{d} \tilde{\phi}-\frac{U(\tilde{\phi})}{2} \tilde{g}_{a b}
$$

is the canonical stress-energy tensor for a scalar field minimally coupled with the curvature, which satisfies the weak energy condition if $\underset{\sim}{V} \geq 0$.

When the scalar field $\tilde{\phi}$ is constant (which only happens if its Jordan frame cousin $\phi$ is constant) then one obtains the same equations of motion as in GR (with a cosmological constant if $U(\tilde{\phi}) \neq 0$, which is equivalent to $V(\phi) \neq 0$, as follows from Eq. (1.9)) .

We are now ready to prove the new no-hair result for scalar-tensor black holes.

\section{JORDAN FRAME NO-HAIR}

Consider a scalar-tensor theory in which the BransDicke coupling is a function $\omega(\phi)$ [27] with a potential $V(\phi)$ and assume spherical symmetry. Up to singular 
exceptions, the most general spherically symmetric line element can be written as

$$
d s^{2}=-A^{2}(t, r) d t^{2}+B^{2}(t, r) d r^{2}+r^{2}\left(d \theta^{2}+\sin ^{2} \theta d \varphi^{2}\right)
$$

in spherical coordinates $(t, r, \theta, \varphi)$. We assume also that the Brans-Dicke scalar is spherically symmetric, $\phi=$ $\phi(t, r)$. The only non-vanishing Christoffel symbols are

$$
\begin{aligned}
& \Gamma_{00}^{0}=\frac{\dot{A}}{A}, \quad \Gamma_{01}^{0}=\Gamma_{10}^{0}=\frac{A^{\prime}}{A}, \quad \Gamma_{11}^{0}=\frac{B \dot{B}}{A^{2}} \\
& \Gamma_{00}^{1}=\frac{A A^{\prime}}{B^{2}}, \quad \Gamma_{01}^{1}=\Gamma_{10}^{1}=\frac{\dot{B}}{B}, \quad \Gamma_{11}^{1}=\frac{B^{\prime}}{B} \\
& \Gamma_{22}^{1}=-\frac{r}{B^{2}}, \quad \Gamma_{33}^{1}=-\frac{r}{B^{2}} \sin ^{2} \theta \\
& \Gamma_{12}^{2}=\Gamma_{21}^{2}=\frac{1}{r}, \quad \Gamma_{33}^{2}=-\cos \theta \\
& \Gamma_{13}^{3}=\Gamma_{31}^{3}=\frac{1}{r}, \quad \Gamma_{23}^{3}=\Gamma_{32}^{3}=\cot \theta
\end{aligned}
$$

Using these quantities and the notation $\cdot \equiv \partial_{t}$ and ${ }^{\prime} \equiv \partial_{r}$ we have, in the geometry (2.1),

$$
\begin{aligned}
\nabla^{c} \phi \nabla_{c} \phi= & -\frac{\dot{\phi}^{2}}{A^{2}}+\frac{\phi^{2}}{B^{2}}, \\
\square \phi= & -\frac{1}{A^{2}}\left(\ddot{\phi}-\frac{\dot{A}}{A} \dot{\phi}-\frac{A A^{\prime}}{B^{2}} \phi^{\prime}\right) \\
& +\frac{1}{B^{2}}\left(\phi^{\prime \prime}-\frac{B \dot{B}}{A^{2}} \dot{\phi}-\frac{B^{\prime}}{B} \phi^{\prime}\right) \\
& +\frac{2 \phi^{\prime}}{r B^{2}} .
\end{aligned}
$$

Since the endpoint of gravitational collapse must be a stationary black hole (cf. [18, 20, 21]), we assume a static scalar field and metric $(\dot{\phi}=0, \dot{A}=\dot{B}=0)$. In electrovacuo or in the presence of conformally invariant matter $1 T^{(m)}=0$ and the d'Alembertian (2.8) reduces to

$$
\square \phi=\frac{1}{B^{2}}\left[\phi^{\prime \prime}+\left(\frac{A^{\prime}}{A}-\frac{B^{\prime}}{B}+\frac{2}{r}\right) \phi^{\prime}\right]
$$

while Eq. (1.3) becomes

$$
\begin{array}{r}
(2 \omega+3)\left[\phi^{\prime \prime}+\left(\frac{A^{\prime}}{A}-\frac{B^{\prime}}{B}+\frac{2}{r}\right) \phi^{\prime}\right] \\
=-\frac{d \omega}{d \phi} \phi^{\prime 2}+B^{2}\left(\phi V_{\phi}-2 V\right),
\end{array}
$$

\footnotetext{
1 This situation includes the Maxwell field, a conformally coupled scalar field with zero or quartic potential, and a radiation fluid.
}

where $V_{\phi} \equiv d V / d \phi$. Since the line element (2.1) is spherically symmetric and is expressed in Schwarzschild-like coordinates employing the areal radius, the apparent horizon is the locus $\nabla_{c} r \nabla^{c} r=0$ [30 33] and it coincides with the event horizon because the geometry is static [34]. The horizon radius $r_{H}$ is given by the (positive) root of $g^{r r}=0$ in these coordinates. The horizon at $g^{11}=0$ is obtained as $B \rightarrow \infty$. To proceed, note that

$$
\nabla^{c} \phi \nabla_{c} \phi=\left(\phi^{\prime}\right)^{2} \nabla^{c} r \nabla_{c} r=\frac{\left(\phi^{\prime}\right)^{2}}{B^{2}}=0
$$

on the horizon, provided that $\phi$ and $\phi^{\prime}$ are well defined there.

Let us assume now, as in the original Brans-Dicke theory [26] and in Hawking's theorem [18] that the potential $V(\phi)$ vanishes identically 2 This is clearly a limitation but it allows one to proceed with the proof. Equation (2.10) then gives

$$
\begin{aligned}
& \frac{\phi^{\prime \prime}}{\phi^{\prime}}+\frac{\omega_{\phi} \phi^{\prime}}{2 \omega+3}+\frac{A^{\prime}}{A}-\frac{B^{\prime}}{B}+\frac{2}{r} \\
= & {\left[\ln \left(\frac{A r^{2} \phi^{\prime}}{B} \sqrt{2 \omega+3}\right)\right]^{\prime}=0, }
\end{aligned}
$$

which integrates to

$$
\frac{\phi^{\prime}(r)}{B}=\frac{C_{0}}{\sqrt{2 \omega+3} A r^{2}},
$$

with $C_{0}$ being an integration constant. Assuming that $\phi$ and $\phi^{\prime}$ are finite on the black hole horizon, the condition $1 / B^{2} \rightarrow 0$ as $r \rightarrow r_{H}$ yields

$$
\frac{C_{0}}{r_{H}^{2} A\left(r_{H}\right)}=0
$$

and the constant $C_{0}$ must vanish. This means that

$$
\phi^{\prime}(r)=0 \quad \forall r \geq r_{H}
$$

and $\phi=$ constant $\forall r \geq r_{H}$. Therefore, the black hole must reduce to a GR black hole. As a conclusion, in the scalar-tensor theory (1.1) and under the assumptions above, a black hole is necessarily a GR black hole. This proof shows that the limitations imposed in the usual theorems are not due to the choice of conformal frame and that it is worth attempting to extend the existing no-hair theorems using the Jordan frame.

\section{CONCLUSIONS}

The proof of the previous section is completed entirely in the Jordan frame. Although the techniques used are

\footnotetext{
2 The same result is obtained if $V(\phi)=m^{2} \phi^{2} / 2$, in which case the potential disappears from the Klein-Gordon-like Eq. (1.3) ruling the dynamics of $\phi$ [35], but not from the field equations (1.2).
} 
different than the usual Einstein frame techniques, the result shows that there is no a priori reason why the Jordan frame should not be useful for this kind of study. The fact that the Jordan frame proof presented cannot be completed when a non-trivial scalar field potential is present is due to purely technical reasons (the fact that it becomes impossible to integrate analytically the nonlinear ordinary differential equation (2.10), and not to reasons of principle. The Jordan frame proof does not require the weak energy condition to be satisfied by the scalar field (in general it is not satisfied, contrary to the Einstein frame), and the proof is restricted to spherically symmetric black holes.

We assumed staticity of the metric and scalar field as features characterizing the endpoint of collapse 3 Strictly speaking, we did not assume asymptotic flatness or de Sitter asymptotics. Removing the assumption of asymptotic flatness present in most no-hair theorems (or that of de Sitter asymptotics) and allowing for more general asymptotics (for example FLRW), would be a major step forward in the existing no-hair theorems. However, we do not claim such a generalization here because 1) it is hard to see how physically significant non-asymptotically flat black holes could arise in vacuo in the absence of a scalar field potential $V(\phi)$ and 2) when the field $\phi$ becomes constant, which happens in both our proof and those of the existing no-hair theorems, the theory reduces to GR but the only possible asymptotics are then constrained to be Minkowski or de Sitter. More interesting situations, for example those involving $f(R)$ black holes, must allow for FLRW asymptotics. As remarked in [11, 20], it is true that one then expects variability on cosmological time scales much larger than the local (astrophysical) time scales, and that these variations could be neglected for the purposes of astrophysics, but one would nevertheless like to have a more complete picture, which is not available at the moment.

\section{ACKNOWLEDGMENTS}

This work is supported by the Natural Sciences and Engineering Research Council of Canada.
[1] L. Amendola and S. Tsujikawa, Dark Energy, Theory and Observations (Cambridge University Press, Cambridge, England, 2003).

[2] E.V. Linder, Am. J. Phys. 76, 197 (2008).

[3] S. Capozziello, S. Carloni, and A. Troisi, Recent Res. Dev. Astron. Astrophys. 1, 625 (2003) arXiv:astro-ph/0303041.

[4] S.M. Carroll, V. Duvvuri, M. Trodden, and M.S. Turner, Phys. Rev. D 70, 043528 (2004).

[5] D.N. Vollick, Phys. Rev. D 68, 063510 (2003).

[6] T.P. Sotiriou and V. Faraoni, Rev. Mod. Phys. 82, 451 (2010); A. De Felice and S. Tsujikawa, Living Rev. Relativity 13, 3 (2010); S. Nojiri and S.D. Odintsov, Phys. Repts. 505, 59 (2011).

[7] E. Berti et al., Class. Quantum Grav. 32, 243001 (2015)

[8] T. Baker, D. Psaltis, and C. Skordis, Astrophys. J. 802, 63 (2015).

[9] W. Israel, Phys. Rev. 164, 1776 (1967); Comm. Math. Phys. 8, 245 (1968); J. Chase, Comm. Math. Phys. 19, 276 (1970); B. Carter, Phys. Rev. Lett. 26, 331 (1971); R.W. Wald, Phys. Rev. Lett. 26, 1653 (1971 ); R. Ruffini and J.A. Wheeler, Phys. Today 24 (1), 30 (1971).

[10] S.W. Hawking, Comm. Math. Phys. 25, 152 (1972).

[11] T.P. Sotiriou, in Modifications of Einstein's Theory of Gravity at Large Distances, Procedings of the Seventh Aegean Summer School, Parikia, Greece, 23-28 September 2013, E. Papantonopoulos ed. (Springer, New York, 2015), pp. 3-33; T.P. Sotiriou, Class. Quantum Grav. 32, 214002 (2015).

\footnotetext{
${ }^{3}$ Staticity of the metric and spherical symmetry do not automatically imply that the scalar field is static, as demonstrated by various scalar field solutions of scalar-tensor gravity [11, 12, 29].
}

[12] C.A.R. Herdeiro and E. Radu, Int. J. Mod. Phys. D 24, 1542014 (2015).

[13] C. Charmousis, in Physics of Black Holes, Procedings of the Fourth Aegean School on Black Holes, Lesvos, Greece, 17-22 September 2007, E. Papantonopoulos ed. (Springer, New York, 2009), pp. 299-343.

[14] J.T. Jebsen, Ark. Mat. Ast. Fys. (Stockholm) 15, nr. 18 (1921), reprinted in Gen. Relat. Gravit. 37, 2253 (2005).

[15] G.D. Birkhoff, Relativity and Modern Physics (Harvard University Press, Cambridge, USA 1923), p. 253

[16] M. Johnson, Lett. Nuovo Cim. 4, 323 (1972). J.D. Bekenstein, Phys. Rev. D 5, 1239 (1972); 5, 2403 (1972); Phys. Rev. Lett. 28, 452 (1972); C. Teitelboim, Lett. Nuovo Cimento 3, 326 (1972); T. Zannias, J. Math. Phys. 36, 6970 (1995); J.D. Bekenstein, Phys. Rev. D 51, R6608 (1995); A. Saa, J. Math. Phys. 37, 2346 (1996); K.A. Bronnikov, Phys. Rev. D 64, 064013 (2001); W. Nelson, Phys. Rev. D 82, 104026 (2010).

[17] J.D. Bekenstein, arXiv:gr-qc/9605059

[18] S.W. Hawking, Comm. Math. Phys. 25, 167 (1972).

[19] V. Faraoni, Phys. Rev. D 81, 044002 (2010).

[20] T.P. Sotiriou and V. Faraoni, Phys. Rev. Lett. 108, 081103 (2012).

[21] S. Bhattacharya, K.F. Dialektopoulos, A.E. Romano, and T.N. Tomaras, Phys. Rev. Lett. 115, 181104 (2015).

[22] T. Matsuda and H. Nariai, Prog. Theor. Phys. 49, 1195 (1973); M. Shibata, K.I. Nakao, and T. Nakamura, Phys. Rev. D 507304 (1994); M.A. Scheel, S.L. Shapiro, and S.A. Teukolsky, Phys. Rev. D 51, 4236 (1995); T. Harada, T. Chiba, K.I. Nakao, and T. Nakamura, Phys. Rev. D 55, 2024 (1997); J. Novak, Phys. Rev. D 57, 4789 (1998); J. Kerimo and D. Kalligas, Phys. Rev. D 58, 104002 (1998); J. Kerimo, Phys. Rev. D 62, 104005 (2000); D.I. Hwang and D.-H. Yeom, Class. Quantum Grav. 27, 205002 (2010). 
[23] R.H. Dicke, Phys. Rev. 125, 2163 (1962).

[24] R.H. Dicke and P.J.E. Peebles, Phys. Rev. Lett. 12, 435 (1964); M. Ferraris, M. Francaviglia, and G. Magnano, Class. Quantum Grav. 5, L95 (1988); 7, 261 (1990); T. Damour and G. Esposito-Farese, Class. Quantum Grav. 9, 2093 (1992); G. Magnano and L. M. Sokolowski, Phys. Rev. D 50, 5039 (1994); V. Faraoni, E. Gunzig, and P. Nardone, Fundam. Cosm. Phys. 20, 121 (1999); Y. Fujii and K. Maeda, The Scalar-Tensor Theory of Gravitation (Cambridge University Press, Cambridge, England, 2003); E.E. Flanagan, Class. Quantum Grav. 21, 417 (2004); R. Catena, M. Pietroni, and L. Scarabello, Phys. Rev. D 76, 084039 (2007); J. Phys. A 40, 6883 (2007); L. Järv, P. Kuusk, and M. Saal, Phys. Rev. D 76, 103506 (2007); V. Faraoni and S. Nadeau, Phys. Rev. $D$ 75, 023501 (2007); L. Järv, P. Kuusk, and M. Saal, Phys. Rev. D 78, 083530 (2008); S. Carloni, E. Elizalde, S. Odintsov, Gen. Relat. Gravit. 42, 1667 (2010); S. Capozziello and D. Saez-Gomez, AIP Conf. Proc. 1458, 347 (2011); P. Creminelli, Phys. Rev. D 85, 041302 (2012); A. Stabile, A. Stabile, and S. Capozziello, Phys. Rev. D 88, 124011 (2013); M. Tsamparlis, A. Paliathanasis, S. Basilakos, and S. Capozziello, Gen. Relat. Gravit. 45, 2003 (2013); P. Kuusk, L. Järv, and O. Vilson, Int. J. Mod. Phys. A 31, 1641003 (2016); L. Järv, P. Kuusk, M. Saal, and O. Vilson Class. Quantum Grav. 32, 235013 (2015); L. Järv, P. Kuusk, M. Saal, and O. Vilson, arXiv:1512.09166, S. Capozziello, L. Fatibene, and S. Garruto, arXiv:1512.08535 M. Herrero-Valea, Phys. Rev. D 93, 105038 (2016); L. Järv, K. Kannike, L. Marzola, A. Racioppi, M. Raidal, M. Rünkla, M. Saal, H. Veermäe, Phys. Rev. Lett. 118, 151302 (2017); S. Baha- monde, S.D. Odintsov, V.K. Oikonomou, and M. Wright, Ann. Phys. 373, 96 (2016).

[25] R.M. Wald, General Relativity (Chicago University Press, Chicago, 1984).

[26] C.H. Brans and R.H. Dicke, Phys. Rev. 124, 925 (1961).

[27] P.G. Bergmann, Int. J. Theor. Phys. 1, 25 (1968); R.V. Wagoner, Phys. Rev. D 1, 3209 (1970); K. Nordvedt, Astrophys. J. 161, 1059 (1970).

[28] K. Nordtvedt, Phys. Rev. 169, 1017 (1968).

[29] E. Ayon-Beato, C. Martinez, R. Troncoso, and J. Zanelli, Phys. Rev. D 71, 104037 (2005); L.M. Sokolowski, Acta Phys. Polon. 35, 587 (2004); E. Ayon-Beato, C. Martinez, and J. Zanelli, Gen. Relat. Gravit. 38, 145 (2006); D.C. Robinson, Gen. Relat. Gravit. 38, 153 (2006); D.A. Demir and B. Pulice, Phys. Lett. B 638, 1 (2006); N. Banerjee, R.K. Jain, and D.P. Jaktar, Gen. Relat. Gravit. 40, 93 (2008); V. Faraoni and A.F. Zambrano Moreno, Phys. Rev. D 81, 124050 (2010); H. Maeda and K.I. Maeda, Phys. Rev. D 86, 124045 (2012).

[30] C.W. Misner and D.H. Sharp, Phys. Rev. 136, B571 (1964).

[31] I. Booth, Can. J. Phys. 83, 1073 (2005).

[32] A.B. Nielsen, Gen. Rel. Gravit. 41, 1539 (2009); Int. J. Mod. Phys. D 17, 2359 (2008).

[33] V. Faraoni, Cosmological and Black Hole Apparent Horizons (Springer, New York, 2015).

[34] S.W. Hawking and G.F.R. Ellis, The Large Scale Structure of Space-Time (Cambridge University Press, Cambridge, England, 1973).

[35] C. Santos, arXiv:gr-qc/0009054. 